\title{
Endoloop-Assisted Alignment-a Simple, Effective, Cheap, and Safe Technique to Aid Specimen Extraction Following Sleeve Gastrectomy
}

\author{
Neil Bhardwaj • Sören T. Mees $\cdot$ Chris Hensman
}

Published online: 11 September 2014

(C) Springer Science+Business Media New York 2014

\begin{abstract}
Keywords Sleeve gastrectomy $\cdot$ Specimen extraction
\end{abstract}

\section{Introduction}

Extraction of the resected gastric specimen following laparoscopic sleeve gastrectomy through a $15-\mathrm{mm}$ port site incision can be tedious, time consuming, and lead to surgeon frustration. The specimen can be bent or torted during the extraction process increasing the risk of tearing and possible contamination of the extraction wound.

In this video, we present a new method of extracting the resected gastric specimen safely and quickly with a reduced risk of contamination of the extraction site.

\section{Method}

After creation of the sleeve gastrectomy, an Endoloop is deployed at the slimmer end of the resected specimen. The end of the loop suture is left intact (without trimming) through

Electronic supplementary material The online version of this article (doi:10.1007/s11695-014-1413-9) contains supplementary material, which is available to authorized users.

N. Bhardwaj $(\bowtie) \cdot$ S. T. Mees

Discipline of Surgery, The Queen Elizabeth Hospital, University of

Adelaide, Woodville, Adelaide 5011, Australia

e-mail: neilbhardwaj@yahoo.co.uk

C. Hensman

LAPSurgery Australia, Boronia, VIC, Australia its delivery port in the left upper quadrant. The specimen is subsequently placed in an Endo Catch II bag (Covidien ${ }^{\circledR}$ ) and sealed. The specimen retrieval bag is delivered via the $15-\mathrm{mm}$ right iliac fossa port and opened outside the anterior abdominal wall. Traction is applied to the end of the Endoloop suture.

Traction on the Endoloop suture aligns the resected gastric specimen to present with its narrowest end promoting easy extraction. Final delivery of the specimen can then be further facilitated by gentle traction with ovum forceps applied directly to the specimen. The specimen bag acts as a wound protector during the extraction process reducing the risks of extraction wound contamination.

\section{Results}

We have used this in over 100 specimen extractions. The technique results in time saving and reduced surgeon frustration.

\section{Conclusion}

The Endoloop-assisted extraction of sleeve gastrectomy specimens is a feasible, economic, and effective way to shorten and facilitate specimen extraction following laparoscopic sleeve gastrectomy. In furthermore, it reduces the risk of port site contamination and reduces surgeon frustration.

\footnotetext{
Disclosures Dr. Neil Bhardwaj has no conflicts of interest or financial ties to disclose.

Dr. Mees has no conflicts of interest or financial ties to disclose.

Dr. Hensman has no conflicts of interest or financial ties to disclose.
} 